\title{
Pediatric Gastropleural fistula, a complication of Sleeve gastrectomy: Case report and brief review.
}

\author{
dana albassam ${ }^{1}$, Mohammed alzaid $^{1}$, Nawaf alotaibi ${ }^{1}$, hammad alsadoon ${ }^{1}$, Felwa alqazlan ${ }^{1}$, \\ and wadha alotaibi ${ }^{1}$ \\ ${ }^{1}$ King Fahad Medical City
}

June 25, 2020

\begin{abstract}
Gastropleural fistula (GPF) is a pathological communication between the stomach and the pleura. Here we describe the first case of pediatric GPF secondary to sleeve gastectomy that was which was managed endoscopically with an over the scope clip (OVESCO) after failure of surgical repair.
\end{abstract}

\section{Key Clinical Message:}

Gastropleural fistula (GPF) is a rare pathological communication between stomach and pleura. It may complicate sleeve gastrectomy. Endoscopic procedure e.g. over the scope clip (OVESCO) can be used to manage GPF.

\section{Introduction:}

Gastropleural fistula (GPF) is a communication between the stomach and pleural that might occur in adult patients as a complication of sleeve gastrectomy (SG) which is a widely accepted weight reduction surgery ${ }^{1,2}$. In our literature review, we found 5 reports of pediatric GPF and none of those cases were related to bariatric weight reduction surgery (Table 1). We report the first pediatric GPF secondary to SG.

\section{Case Description:}

Our patient is an 11 years old boy who underwent SG due to morbid obesity (BMI=55.6). Surgery took place in a private hospital outside the country. It was reported as uneventful and the patient was discharged after 1 week with a nutritional plan and vitamin supplements. Postoperatively, he had no complaints apart from one admission to his local hospital for gastroenteritis and dehydration that was treated with IV fluids and antibiotics.

Five months after his operation, the patient developed fever, followed by productive cough and dyspnea. He was admitted to the local hospital and managed as a case of community acquired pneumonia (CAP) based on the chest x-ray (CXR) findings of localized left lower lobe opacity. A barium meal at that time was reported to be normal. He was not improving, so he was referred to his primary surgeon who did a CT chest with oral contrast which showed a left lower lobe abscess measuring $5 \times 6 \mathrm{~cm}$ and a leak of contrast to the pleural space indicating the presence of a GPF. They elected to manage him with laparoscopic surgery for fistula closure and left lower lobe lobectomy via thoracotomy.

The patient was still having a chronic cough and fluctuating fever, a follow-up barium meal showed a leak at the site of the fistula. And a repeated chest CT scan revealed multiple encysted pockets of fluid within 
the pleura (Figure 1). These thoracic complications were treated with video assisted thoracoscopic surgery (VATS) for decortication with chest tube insertion and to prevent further injury, gastrojeujonal (GJ) tube was inserted for feeding with a second tube introduced through the fistula opening for drainage.

The patient was then referred to our tertiary center to further manage his persistent GPF. He was having a history of fever and chronic dry cough. Upon admission, he was barely reactive due to a depressive state which mandated a psychiatric evaluation; however, he didn't require any medication. His initial lab work-up showed leukocytosis and elevated inflammatory markers. He was managed with empirical broad-spectrum antibiotics covering anaerobes and kept NPO with strict GJ feeding. He then underwent an endoscopy which showed a $5 \mathrm{~mm}$ fistula opening in the upper part of the stomach. An over the scope clip (OTSC) was applied to close the fistula (Figure 2). He had no post procedural complication and was discharged on GJ feeding and a follow up to ensure that there is no persistent leak.

Four weeks after endoscopy, barium meal showed no evidence of leak into the pleura and a follow-up CXR displayed marked improvement in the aeration of the left lung (Figure 1). A repeated CT scan with oral contrast at 8 weeks post endoscopy showed no leak and the pockets of encysted pleural fluid had regressed (Figure1). Informed consent was obtained from the patient's family. Currently, he is completely recovered and tolerating oral feeding.

\section{Discussion:}

GPF is a rare, pathological communication between the stomach and the pleural cavity ${ }^{1}$. It was first described by Markowitz and Herter in 1960 as a complication of hiatal hernia rupture ${ }^{3}$. Since then, it has been reported secondary to trauma, peptic ulcer disease, malignancy (lymphoma and gastric tumors), and due to the spread of infection from adjacent structures such as subdiaphragmatic abscess, empyema, or invasive fungal infection in immunocompromised patients. Recently, with the emerging of obesity related bariatric surgeries, GPF has been documented as a complication of such surgeries in adult patients ${ }^{4}$. One of those surgeries is SG which has been utilized in pediatric patients to a lesser extent ${ }^{2}$. Our patient had SG which was unfortunately complicated by GPF.

GPF has a latency in presentation, presenting months to years after the $\mathrm{SG}^{1}$. The symptoms range from chronic cough to recurrent respiratory infections, both of which were seen in our case ${ }^{1}$. Other respiratory symptoms include chest pain and hemoptysis ${ }^{5}$. Patients might exhibit gastrointestinal symptoms such as hematemesis or abdominal pain ${ }^{16}$. GPF can be complicated by sepsis, hydropneumothorax, or tension pneumothorax. Chest tube drainage containing food particles or bile has also been reported ${ }^{4}$.

Diagnosis of GPF requires a high index of suspicion and thorough investigations ${ }^{4}$. Initial workup includes CXR, which may demonstrate pneumothorax, pleural effusion, or hydropneumothorax ${ }^{4}$. Upper GI series, preferably with water-soluble contrast, might show contrast extravasation to the pleural cavity ${ }^{6}$. CT with oral contrast may reveal contrast leakage into the pleural space or delineate the fistula tract $^{4}$. Moreover, upper GI endoscopy may identify the site of the fistula and can be utilized for therapeutic purposes ${ }^{7}$. Occasionally, GPF is diagnosed intraoperatively ${ }^{8}$.

There are no standardized treatment protocols for $\mathrm{GPF}^{7}$. Initial steps of management consist of treating the GPF complications and providing an alternative to oral feeding (parenteral nutrition or jejunostomy feeding tube $)^{7,5}$. Meanwhile, the closure of the fistula can be done endoscopically or surgically. Various endoscopic interventions have been used including endoscopic clips stents, suturing, and fistula plug application ${ }^{7,9,10}$. Our patient was managed conservatively and the fistula was closed endoscopically with an OTSC.

OTSC is a relatively new endoscopic intervention that has been used for chronic fistulas with variable success rate, ranging from $30 \%$ to $100 \%$, and a low complication rate. Other indications for OTSC include gastrointestinal hemorrhage and perforation ${ }^{11}$. Our patient failed surgical management, (i.e. trial of fistula closure laparoscopically). Other surgical options include partial gastric resection, diaphragmatic repair, and washout and drainage of the chest ${ }^{4}$. Roux-en-Y esophagojejunostomy for chronic GPF has also been used for chronic fistula ${ }^{5}$. In conclusion, pediatric GPF has been reported due to various causes (table1). Our 
patient is the first report of pediatric GPF secondary to SG. SG is not without its complications and it must be reserved for morbidly obese patients who fail conservative management. In our case, endoscopic intervention with an OTSC had better results when compared to the invasive surgical closure.

Authors contribution:

Author 1: literature review, discussion writing

Author 2: writing case description, discussion writing

Author 3: literature review, discussion writing

Author 4: editing and reviewing case description and discussion

Author 5: writing case description

Author 6: editing and reviewing case description and discussion

Conflict of interest:

None declared

References:

1. Alghanim Fahad, Alkhaibary Ali AA and AA. Had Be j. Case Rep Surg . 2018;2018.

2. Alqahtani A, Alamri H, Elahmedi M, Mohammed R. Laparoscopic sleeve gastrectomy in adult and pediatric obese patients: A comparative study.Surg Endosc . 2012;26(11):3094-3100. doi:10.1007/s00464012-2345-x

3. Markowitz AM, Herter FP. Gastro-pleural fistula as a complication of esophageal hiatal hernia. Ann Surg . 1960;152:129-134. doi:10.1097/00000658-196007000-00018

4. Armstrong RL, Heyse PB. Gastropleural fistula in metastatic ovarian cancer. J Surg Case Rep . 2014;2014(5):rju033-rju033. doi:10.1093/jscr/rju033

5. Garcia-Quintero P, Hernandez-Murcia C, Romero R, Derosimo J, Gonzalez A. Gastropleural fistula after bariatric surgery: a report of two cases.J Robot Surg . 2015;9(2):163-166. doi:10.1007/s11701-015-0505-4

6. Tzeng JJ, Lai KH, Lo GH, Hsu JH, Mok KT. Gastropleural fistula caused by incarcerated diaphragmatic herniation of the stomach. Gastrointest Endosc . 2001;53(3):382-384. doi:10.1067/mge.2001.111563

7. Ghanem OM, Abu Dayyeh BK, Kellogg TA. Management of Gastropleural Fistula after Revisional Bariatric Surgery: A Hybrid Laparoendoscopic Approach. Obes Surg . 2017;27(10):2773-2777. doi:10.1007/s11695-017-2836-x

8. Lakshminarayanan B, Morgan RD, Platt K, Lakhoo K. A leak too far - Gastro-pleural fistula mimicking recurrence of repaired congenital diaphragmatic hernia following fundoplication. J Radiol Case Rep . 2013;7(9):33-38. doi:10.3941/jrcr.v7i9.1505

9. Mendoza Ladd A, Al-Bayati I, Shah P, Haber G. Endoscopic closure of a gastropleural fistula. Endoscopy . 2015;47:E131-E132. doi:10.1055/s-0034-1391357

10. Andrawes S, El Douaihy Y. Using the endoscopic overstitching device and fully covered esophageal stents for closure of a gastropleural fistula and repair of a deformed gastric sleeve. VideoGIE . 2017;2(5):98-99. doi:10.1016/j.vgie.2017.02.003

11. Weiland T, Rohrer S, Schmidt A, et al. Efficacy of the OTSC System in the treatment of GI bleeding and wall defects: a PMCF meta-analysis.Minim Invasive Ther Allied Technol . 2019. doi:10.1080/13645706.2019.1590418 
12. Prasertsan P, Anuntaseree W, Ruangnapa K, Saelim K. Gastropleural fistula masquerading as chylothorax in a child with lymphoma. BMJ Case Rep . 2019;12(7):10-13. doi:10.1136/bcr-2018-228987

13. Wuthisuthimethawee P, Sangkhathat S, Ruegklinag C, Patrapinyokul S, Laoprasopwathana K. Gastropleural fistula following a splenectomy for splenic abscess: A case report. J Med Assoc Thai . 2008;91(8):1291-1295.

14. Arun S, Lodha R, Sharma R, Agarwala S, Kabra SK. Gastropleural fistula as a complication of empyema thoracis. Indian J Pediatr . 2007;74(3):301-303. doi:10.1007/s12098-007-0051-9

15. Malik S, Giacoia GP. Candida Tropicalis Empyema Associated with Acquired Gastropleural Fistula in a Newborn Infant. Am J Perinatol . 1989;6(3):347-348. doi:10.1055/s-2007-999611

Figure 1: This figure shows the improvement in radiological findings. Pre-endoscopic chest $\mathrm{x}$ ray (A) and CT scan (C) which shows a contrast leak and loculated encysted fluid (Star). And post-endoscopic CXR (B) and CT scan with oral contrast (D) which shows a regression of the loculated fluid and no more leak from the fistula.

Figure 2: Endoscopic application of OVESCO

Table 1: Reported GPF in pediatric population

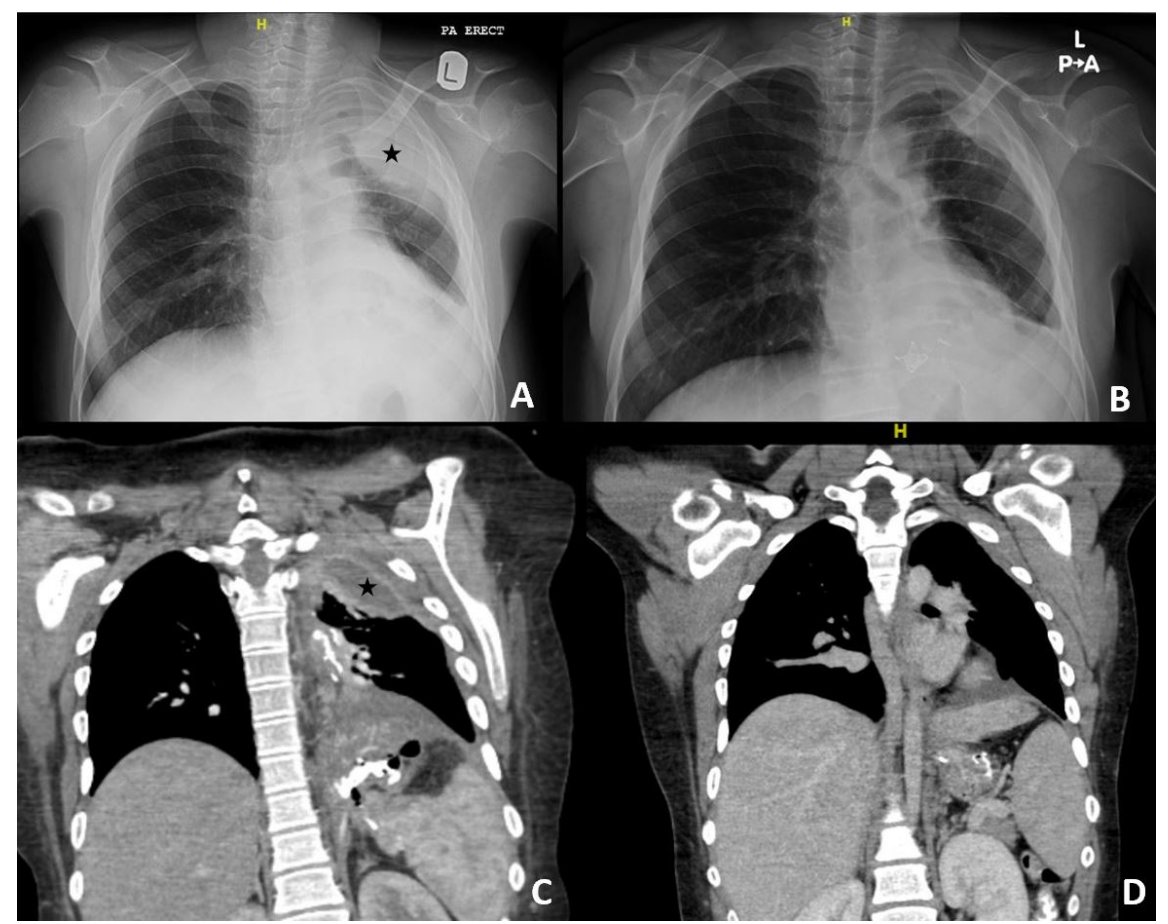




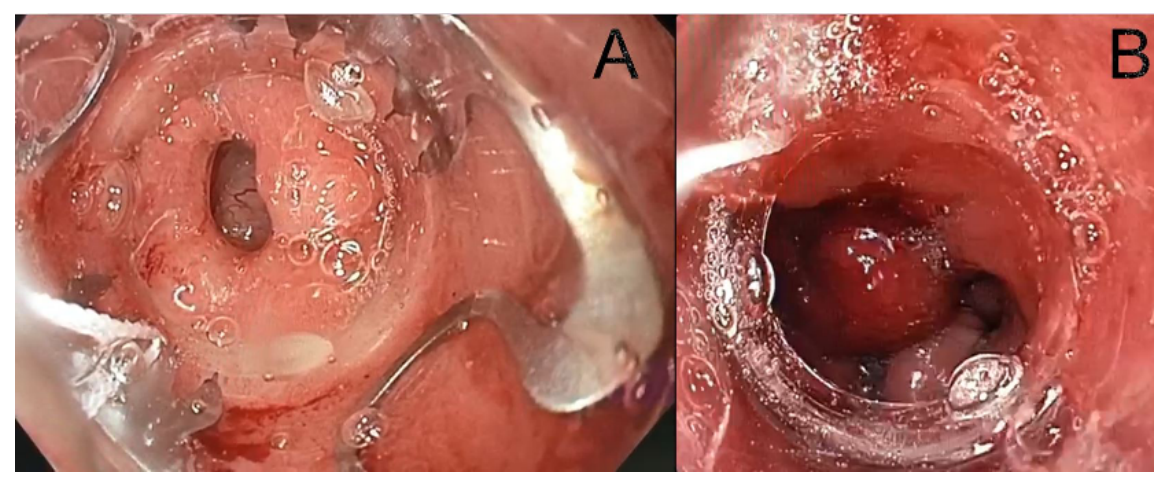

